\title{
Hysteresis-free DC conduction in zinc oxide films with a conducting polymer counter electrode
}

\author{
Sharmistha Paul ${ }^{1}$ - Paul G. Harris ${ }^{2}$ - Ashwani K. Sharma ${ }^{3} \cdot$ Asim K. Ray $^{4}$ \\ Received: 5 October 2017 / Accepted: 6 November 2017 / Published online: 15 November 2017 \\ (c) The Author(s) 2017. This article is an open access publication
}

\begin{abstract}
A conducting polymer, namely poly (3,4-ethylenedioxythiophene):poly(styrenesulfonate) (PEDOT:PSS) has been employed as an alternative metal counter electrode to study steady state charge conduction through a spin-coated $5.2 \mu \mathrm{m}$ thick $\mathrm{ZnO}$ film in a sandwich structure on a fluorine doped tin oxide coated glass substrate. The room temperature current-voltage characteristics exhibit rectifying behaviour without hysteresis as the bias voltage is cycled between $\pm 10 \mathrm{~V}$ for varying voltage sweep rate from 100 to $1000 \mathrm{mV} / \mathrm{s}$. Thermionic emission is believed to be the dominant conduction mechanism at forward bias voltage $\mathrm{V} \leq 2.5 \mathrm{~V}$ whereas the space charge limited current conduction becomes effective in a higher voltage region $\mathrm{V}>2.5 \mathrm{~V}$. The barrier height $\left(\varphi_{b}\right)$, ideality factor $(\mathrm{n})$ and series resistance $\left(\mathrm{R}_{\mathrm{s}}\right)$ are found to be strongly temperature dependent parameters showing the increase of $\phi_{b}$ and simultaneous decreases of $n$ and $\mathrm{R}_{\mathrm{s}}$ within the range of 218-298 K. The behaviour of the temperature dependent charge carrier mobility in the higher voltage region has also been discussed. The use of PEDOT:PSS is quite promising as an alternative to metal electrodes in semiconductor devices.
\end{abstract}

\section{Introduction}

The optical, electrical/electronic properties and stability of intrinsic conducting polymers are now considered to be sufficiently good for their use as alternatives to costly metal electrodes in the low cost production of high volume, large area, and flexible transparent electronic devices [1]. The $p$-type conducting polymer, polypyrrole has beeen used to fabricate a Schottky contact of high quality on $n$-type inorganic compound semiconductors devices such as indium phosphide (InP) [2]. In recent years, the conducting polymer poly (3,4-ethylenedioxythiophene):poly(styrenesulfon ate) (PEDOT:PSS), which has HOMO and LUMO level at 5.2 and $3.5 \mathrm{eV}$ respectively, is being increasingly employed

Asim K. Ray

asim.Ray@ rhul.ac.uk

1 West Bengal State Council of Science \& Technology, Vigyan Chetana Bhavan, 26/B, DD Block, Sector1, Salt Lake, Kolkata 700064, India

2 Institute of Materials and Manufacturing, Brunel University, Uxbridge, Middlesex UB8 3PH, UK

3 United States Air Force Research Laboratory, Space Vehicles Directorate, SE Kirtland AFB, NM 87117, USA

4 Royal Holloway, University of London, Egham, Surrey TW20 0EX, UK as a hole injecting electrode in organic devices because of its high electrical conductivity which can be as large as $4380 \mathrm{~S} /$ $\mathrm{cm}$, high optical transparency of nearly $90 \%$ and low cost large volume solution processability [3-5]. $200 \mathrm{~nm}$ thick spin coated PEDOT:PSS films are found to exhibit electrochromically fast response within $5 \mathrm{~s}$ for colour switching under the voltage cycling between $\pm 3.5 \mathrm{~V}$ [6]. Unlike indium tin oxide (ITO) electrodes, PEDOT:PSS electrode is environmentally friendly and the peak power efficiency of organic light emitting devices using multi-layered PEDOT:PSS electrode are reported to be $44 \%$ greater than those with ITO anodes without any outcoupling-enhancing structures [7]. The average field effect hole mobility is estimated to be $0.157 \mathrm{~cm}^{2} \mathrm{~V}^{-1} \mathrm{~s}^{-1}$ for bottom gate organic thin film transistors using pentacene film as the main channel and electrohydrodynamic-printed PEDOT:PSS drain and source. This value is three orders of magnitude higher than the one obtained from similar pentacene transistors with vacuum deposited gold ( $\mathrm{Au}$ ) source and drain electrodes. Additionally, the resistances at PEDOT:PSS contacts is reported to be smaller than at Au contacts [8]. Semi-transparent, all plastic non-fullerene organic cells using film-transfer laminated PEDOT:PSS as the top electrode, are reported to exhibit $50 \%$ average visible transparency, meeting the requirement of the requirement of electricity generating windows and the power conversion efficiency is better than fullerene-based 
cells with similar thickness and transparency [9]. Highly flexible, stable, transparent supercapacitors have been fabricated using aerosol-jet sprayed sulfuric acid-polyvinyl alcohol $\left(\mathrm{H}_{2} \mathrm{SO}_{4} / \mathrm{PVA}\right)$ gel electrolytes as the binder and separator, sandwiched between two PEDOT:PSS electrodes. The devices with capacitance/areas of $0.84 \mathrm{mFcm}^{-2}$ were found to be optically $80 \%$ transparent and able to endure 10,000 galvanostatic charge/discharge cycles at $20 \mathrm{~A} / \mathrm{m}^{2}$ [10].

Efforts have been made to produce good electrical contacts, Ohmic and Schottky, on zinc oxide ( $\mathrm{ZnO})$, a low toxicity, n-type oxide semiconductor having direct wide band gap of $3.37 \mathrm{eV}$ and large electron affinity of $4.1-4.5 \mathrm{eV}$, for realising its many potential applications in flexible, transparent electronics [11]. $50 \mathrm{~nm}$ thick spin-coated PEDOT:PSS films on a single crystal $\mathrm{ZnO}$ (0001) is reported to have produced excellent Schottky junctions with a high rectifying ratio of $10^{10}$ at $\pm 2 \mathrm{~V}$, low leakage current of $10 \mathrm{pA}$ up to $-10 \mathrm{~V}$ and an ideality factor of 1.2. These results are comparable to those obtained for Schottky junctions with platinum and gold electrodes [12]. This type of Schottky diode can be employed as a highly sensitive ultraviolet detector with unity quantum efficiency. Values of $3.6 \times 10^{14} \mathrm{cmHz}^{1 / 2} \mathrm{~W}^{-1}$ and $10^{3}$ have been estimated for normalised detectivity at $370 \mathrm{~nm}$ and the rejection ratio at zero bias condition, respectively [13]. The problem associated with the chemical activity between PEDOT:PSS electrodes and epitaxial grown $\mathrm{ZnO}$ films with O-polar surfaces can also be overcome by simply treating film surfaces with a silane-coupling agent of 3-aminopropyltriethoxysilane [14].

Low cost, room temperature printing techniques for depositing $\mathrm{ZnO}$ thin films on fluorine-doped tin oxide (FTO) substrate have been developed by us using commercially available zinc oxide $(\mathrm{ZnO})$ nanoparticles. The performance of two terminal FTO/ZnO/Au memresistors devices depends upon the voltage sweep rate, showing on-off ratios as large as $10^{4}$ for $100 \mathrm{mV} / \mathrm{s}$ [15]. Multiple hopping between trap sites within the grain and grain boundary regions are believed to be responsible for charge transport through the FTO/ZnO/Au sandwich structure [16].

In this study, charge transport mechanisms of $\mathrm{FTO} / \mathrm{ZnO} /$ PEDOT:PSS device have been determined through careful analysis of current-voltage (I-V) characteristics measured over a temperature range between 220 and $300 \mathrm{~K}$ when the bias voltage $\mathrm{V}$ was cycled between $\pm 10 \mathrm{~V}$ at three different rates of 100,500 and $1000 \mathrm{mV} / \mathrm{s}$. Comparison with FTO/ $\mathrm{ZnO} / \mathrm{Au}$ devices is made to evaluate the significance of using PEDOT:PSS as a metal alternative electrode material. Electrical parameters on Schottky diode using RF sputtered $\mathrm{ZnO}$ film on the ITO substrate and PEDOT:PSS have recently been reported over the limited bias voltage range of $\pm 1 \mathrm{~V}$ at higher temperatures varying between 302 and $373 \mathrm{~K}$ [17].

\section{Experimental}

Ultrasonically cleaned glass slides coated in fluorine-doped tin oxide (FTO) with a nominal surface resistivity of $\sim 12$ $\Omega /$ sq (supplied by VisionTek Systems, UK) were used for this investigation. The relatively less expensive FTO-coated substrates were chosen as the substrates for the $\mathrm{ZnO}$ film deposition because of their high conductivity, of the order of $2.3 \mathrm{Sm}^{-1}$, excellent chemical stability and $80 \%$ optical transparency within the visible region [18]. Thin films of $\mathrm{ZnO}$ were deposited on FTO-coated substrates by spinning a small volume of a $40 \mathrm{wt} \%$ dispersion of $\mathrm{ZnO}$ nanoparticles $(<130 \mathrm{~nm})$ in ethanol (obtained from Sigma-Aldrich) at 250 and $2000 \mathrm{rpm}$ sequentially for 15 and $60 \mathrm{~s}$ respectively. The solvent was then completely removed by oven drying the films in air at $120^{\circ} \mathrm{C}$ for $15 \mathrm{~min}$. The samples were annealed at $500{ }^{\circ} \mathrm{C}$ in air for $2 \mathrm{~h}$ through $30 \mathrm{~min}$ long temperature ramping from room temperature and back. The choice of the substrates depends upon the type of the measurement undertaken.

$\mathrm{X}$-ray diffraction $(\mathrm{XRD})$ patterns were acquired for the $\mathrm{ZnO}$ film on FTO substrates using a Bruker D8 Advance sweeping in the $2 \theta$ range of $5^{\circ}-100^{\circ}$, with a step size of $0.02^{\circ} / \mathrm{s}$ using $\mathrm{CuK} \alpha$ radiation of wavelength $0.15406 \mathrm{~nm}$. Photoluminescence excitation and emission spectra of $\mathrm{ZnO}$ film on quartz substrates were measured at room temperature in the range from 250 to $800 \mathrm{~nm}$ by Horiba Jobin Yvon Fluorolog-3 spectrofluorometer with a pulsed $450 \mathrm{~W}$ Xenon lamp. Fourier transform infrared spectroscopy (FTIR) of the same film was recorded in the range of $400-4000 \mathrm{~cm}^{-1}$ at a resolution of $4 \mathrm{~cm}^{-1}$ by using Perkin Elmer Spectrum One FTIR spectrometer at room temperature.

For electrical characterisation, PEDOT:PSS polymer (Orgacon 3400, Agfa Speciality Products) was used as the top electrode of the $5.2 \mu \mathrm{m}$ thick $\mathrm{ZnO}$ film in a sandwich configuration with FTO as the bottom electrode. PEDOT:PSS was spin-coated at $1000 \mathrm{rpm}$ for $60 \mathrm{~s}$ on annealed $\mathrm{ZnO}$ films. The solution processing method of depositing the PEDOT:PSS electrode was chosen in order to avoid the possible formation of interfacial trap states. A Keithley 617 electrometer and an Oxford Instruments constant temperature liquid nitrogen cryostat were used in a microprocessor controlled measuring system to record steady state current-voltage characteristics for $\mathrm{ZnO}$ sandwich devices in vacuum of $10^{-4}$ mbar over the temperature range of 218-298 K. The voltage was applied to the sample through the PEDOT:PSS electrode from -10 to $+10 \mathrm{~V}$ with scan rate $500 \mathrm{mV} / \mathrm{s}$ keeping the FTO electrode grounded. 


\section{Results and discussion}

Figure 1 shows the XRD pattern of the annealed $\mathrm{ZnO}$ spun film on FTO substrates. The peaks at $31.81^{\circ}, 34.41^{\circ}, 36.24^{\circ}$, $47.51^{\circ}, 56.62^{\circ}, 62.86^{\circ}, 66.40^{\circ}, 69.10^{\circ}$ and $72.55^{\circ}$ are identified with diffraction at (100), (002), (101), (102), (110), (103), (200) (112) and (004) planes because of their agreement with the reference values of JCPDS (36-1451). The intensity ratio $I_{(002)} / I_{(101)}$ is estimated to be nearly 0.5 which is close to the typical value of 0.44 for the bulk hexagonal wurtzite structure. A plot in the inset of Fig. 1 of $\beta \cos \theta$ as a function of $\sin \theta$ is found to be linear showing the validity of the Williamson-Hall equation in the form [19]:

$\beta \cos \theta=\eta \sin \theta+\frac{\lambda}{D}$

where $\beta$ is the angular line width of half maximum intensity at the Bragg angle $\theta$ and $\lambda=0.1540 \mathrm{~nm}$. Values of $28.36 \mathrm{~nm}$ and 0.01 are found for the average crystallite size $D$ and the strain $\eta$ from the intercept and the slope of the linear plot. These XRD results indicate the formation of an improved structure of $\mathrm{ZnO}$ films over ones recently reported for chemical bath deposited $\mathrm{ZnO}$ films [20]. The intensity ratio $I_{(002)} / I_{(101)}$ of 0.70 is considerably higher while a value of $22 \mathrm{~nm}$ for the average crystallite size $D$ is smaller than the respective values obtained in the present investigation.

Figure 2 presents the mid-infrared regime FTIR spectrum for the annealed $\mathrm{ZnO}$ film between 1000 and $4000 \mathrm{~cm}^{-1}$. The broad peak around $3365 \mathrm{~cm}^{-1}$ corresponds to the $\mathrm{OH}$ stretching vibration, implying the possible presence of residual hydroxyl ions in the $\mathrm{ZnO}$ film. The occurrence of this peak at a similar position has been reported for the $\mathrm{ZnO}$ film, newly synthesised by a wet chemical method using

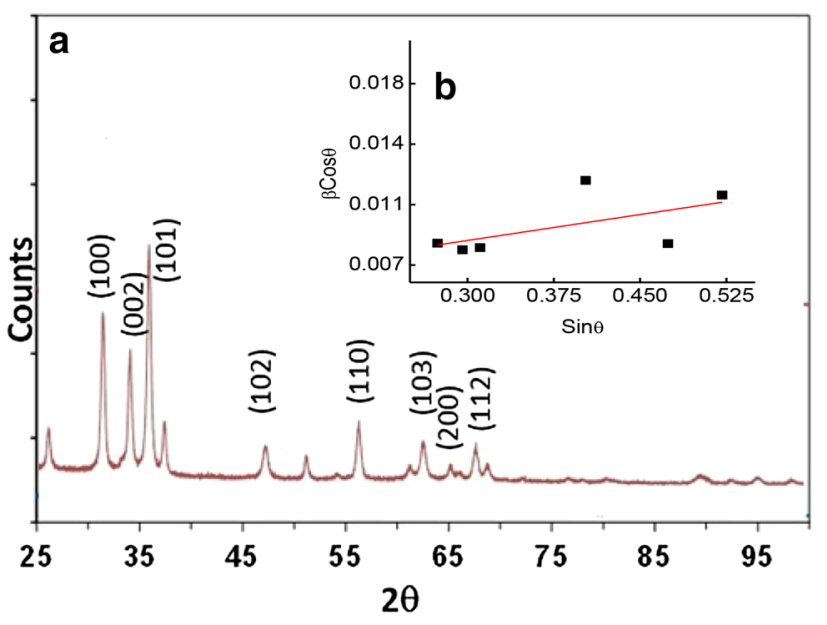

Fig. 1 a XRD pattern of annealed $\mathrm{ZnO}$ film on FTO coated glass slide. b Plot of William-Hull line for the sample

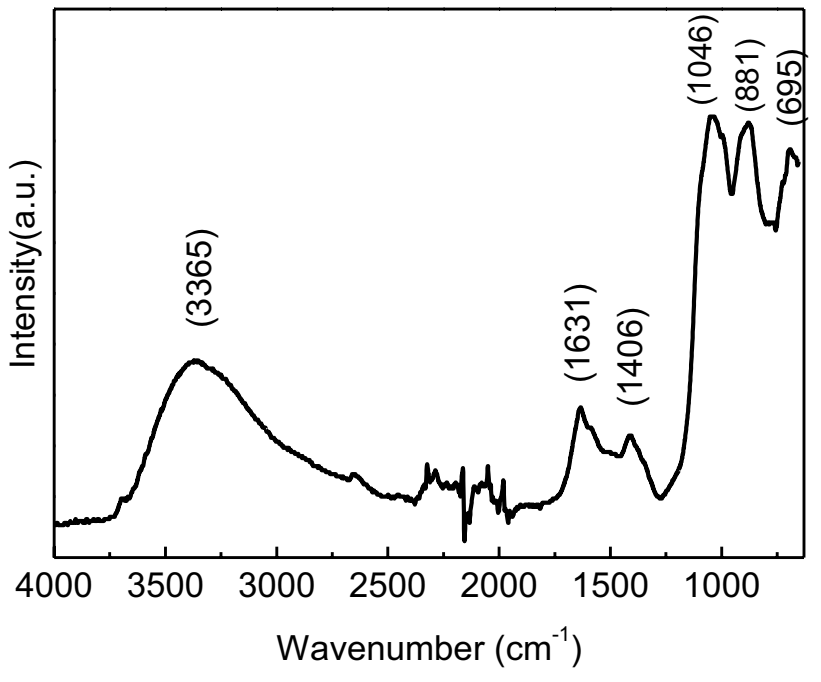

Fig. 2 FTIR spectra of annealed ZnO film on FTO coated glass slide

triethanolamine as a mild base [21]. The presence of two peaks at 1631 and $1406 \mathrm{~cm}^{-1}$ may be attributed to asymmetric and symmetric $\mathrm{C}-\mathrm{O}$ stretching. The peaks at 881 and $695 \mathrm{~cm}^{-1}$ are due to $\mathrm{Zn}-\mathrm{O}$ stretching [22].

The room temperature photoluminescence (PL) emission and excitation spectra of annealed $\mathrm{ZnO}$ film are shown in Fig. 3. The observation of a strong luminescence peak at $395 \mathrm{~nm}$ corresponding to $3.14 \mathrm{eV}$ in the UV region of the emission spectra may be identified with near band energy emission and is in agreement with the value reported for ultrasonic spray pyrolysis deposited $\mathrm{ZnO}$ films on an FTO substrate [23]. The origin of this peak is usually attributed to the recombination of free excitons through exciton-exciton

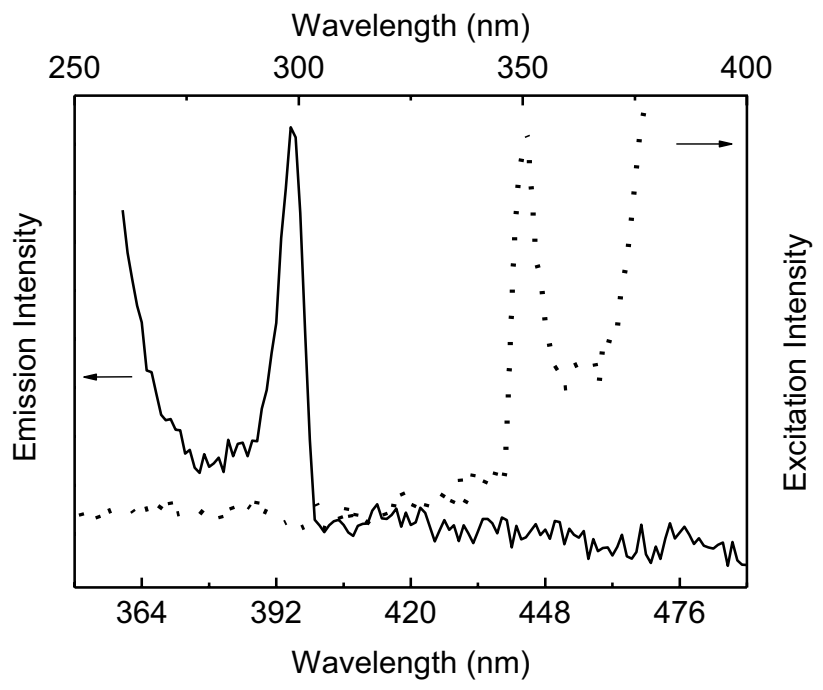

Fig. 3 Photoluminescence spectra of annealed $\mathrm{ZnO}$ film: emission (solid line). Excitation spectra (broken line). Arrows show the relevant axes 
collision process associated with the transition over the wide direct band gap of $\mathrm{ZnO}$ from the ground state to the continuum state [24]. No pronounced peak was found to have occurred in the visible range of the emission spectrum. This absence implies the formation of high crystal quality of $\mathrm{ZnO}$ films without the significant presence of oxygen vacancies [25]. The PL excitation spectrum is also recorded at the emission wavelength of $395 \mathrm{~nm}$. The occurrence of the sharp band edge peak at $351 \mathrm{~nm}$ of the excitation spectrum may be considered as evidence of good crystalline and high purity synthesized $\mathrm{ZnO}$ nanoparticles. The presence of this strong absorption peak at $351 \mathrm{~nm}$ is considered to be blue-shifted relative to the peak at $373 \mathrm{~nm}$ for the bulk $\mathrm{ZnO}$ corresponding to a small increase of the band gap to $3.53 \mathrm{eV}$ from the bulk value of $3.32 \mathrm{eV}$ [26].

Figure 4 a presents a set of reproducible room temperature current-voltage $(I-V)$ curves of a FTO/ZnO/PEDOT:PSS structure as depicted in the top inset for one complete cycle between $\pm 10 \mathrm{~V}$, as bias voltage $\mathrm{V}$ was swept in both forward and reverse directions through $0 \mathrm{~V}$ at three different sweep rates namely 100,500 and $1000 \mathrm{mV} / \mathrm{s}$ respectively. The
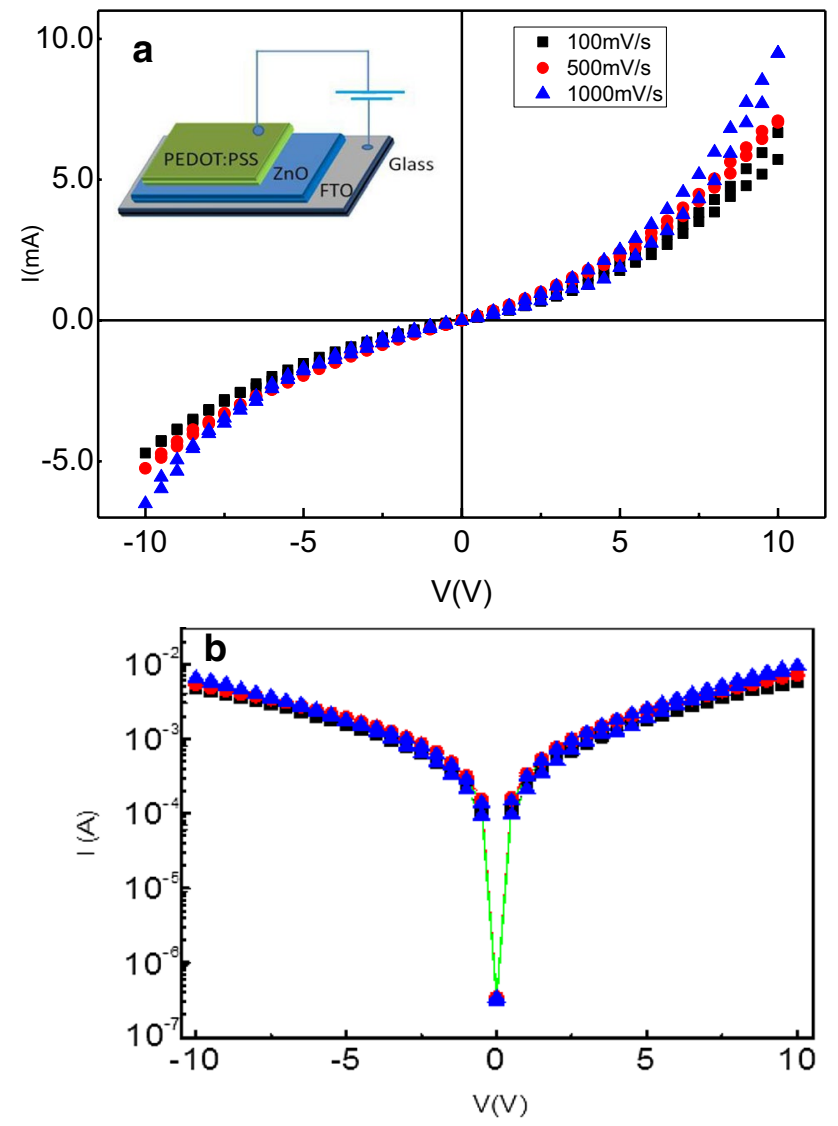

Fig. 4 a One complete cycle of $I-V$ measurement of $\mathrm{FTO} / \mathrm{ZnO} /$ PEDOT:PSS at different voltage sweep rates. Insets showing the device sandwich structure. b Plot of $I-V$ on a logarithm-linear scale for the same device magnitude of current in both bias directions is three orders higher than one reported for the similarly fabricated FTO/ $\mathrm{ZnO} / \mathrm{Au}$ device which showed multi-level conducting states in their (I-V) with large on-off ratios [15]. This implies that the PEDOT:PSS is more efficient in charge injection into the $\mathrm{ZnO}$ film than the Au electrode. The semi-logarithmic plot of $I-V$ characteristics in Fig. $4 \mathrm{~b}$ clearly indicates the absence of hysteresis for the measured sweep rates. The self-healing breakdown which is regarded as being responsible for existence of multi-level resistance states in the $\mathrm{I}(\mathrm{V})$ characteristics for the structure with an Au counter electrode seems not occur in FTO/ZnO/PEDOT:PSS device [27]. Further, the current $\mathrm{I}$ is found to have increased linearly at bias voltage $\mathrm{V} \leq 1 \mathrm{~V}$ for all three sweep rates. However, the dependence of I on $\mathrm{V}$ becomes non-linear for $\mathrm{V}>1 \mathrm{~V}$. The voltage-dependent rectification ratio is found to lie within two orders of magnitude for different voltage sweep rates for the device. For the voltage region higher than $2.5 \mathrm{~V}$, $\mathrm{m}$ is found to vary between 1.5 and 2 , indicating that space-charge limited current (SCLC) conduction is occurring, where traps play a major role in controlling performance [28]. Values of $m$ do not show significant variation with the sweep rates. The dependence of current on applied bias and its rectifying nature demonstrate that the PEDOT:PSS layer is behaving as a non-interacting conductive electrode for this device.

Electrical parameters such as barrier height $\left(\phi_{b}\right)$, ideality factor $(n)$, series resistance $\left(R_{\mathrm{S}}\right)$ for Schottky contact between $\mathrm{ZnO} / \mathrm{PEDOT}: \mathrm{PSS}$ may be evaluated by applying thermionic emission theory in lower voltage region I $(0-2.5 \mathrm{~V})$. Therefore, for the applied voltage $V>3 \mathrm{kT} / \mathrm{q}$, the forward bias current at temperature $\mathrm{T}$ can be expressed as [29]:

$I=I_{s}\left[\exp \left(\frac{q\left(V-I R_{s}\right)}{n k T}\right)-1\right]$

where $I_{\mathrm{s}}, R_{\mathrm{s},} q, k$ and $n$ are reverse saturation current, series resistance, elementary charge, Boltzmann constant and ideality factor, respectively. The reverse saturation current $I_{s}$ is written in following form:

$I_{s}=A A * T^{2} \exp \left(\frac{-q \phi_{b}}{k T}\right)$

where $A$ is the active contact area $\left(110 \times 10^{-6} \mathrm{~m}^{2}\right), A^{*}$ is the Richardson constant, which has a theoretical value of $32 \mathrm{~A} \mathrm{~cm}^{-2} \mathrm{~K}^{-2}$ for $\mathrm{ZnO}[30]$ and $\phi_{b}$ is the Schottky barrier height. The value of $I_{\mathrm{s}}$ is obtained from the extrapolated intercept of $\ln I$ versus $V$ plot (Fig. 5) with the current axis for $V=0 \mathrm{~V}$. The calculated barrier heights $\phi_{b}$ at zero bias for different temperatures $(298-218 \mathrm{~K})$ are obtained within the range of $0.29-0.19 \mathrm{eV}$. The expected barrier height at a $\mathrm{ZnO} / \mathrm{PEDOT}$ :PSS junction is $0.7 \mathrm{eV}$ as the HOMO level of 


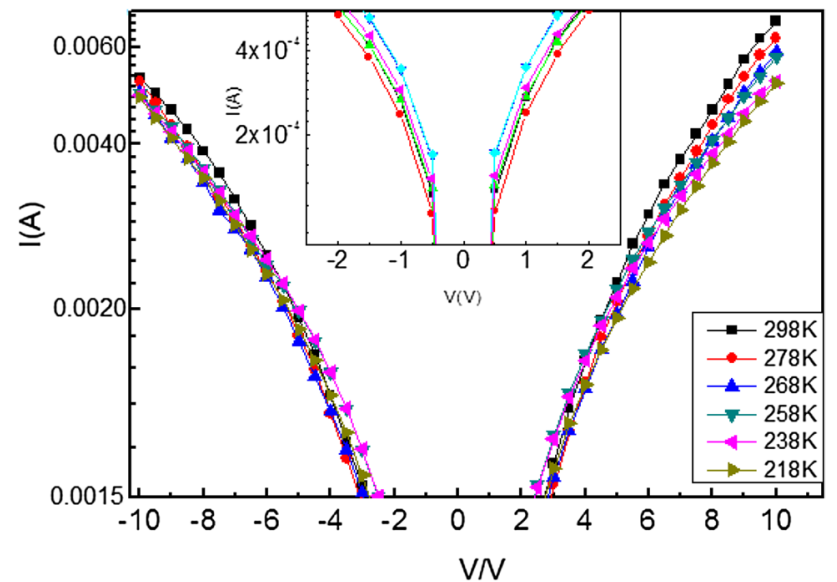

Fig. 5 Semi logarithm plots of $I-V$ curves of FTO/ZnO/PEDOT:PSS device at different temperatures $(218-298 \mathrm{~K})$ between $\pm 10 \mathrm{~V}$ at $500 \mathrm{mV} / \mathrm{s}$ voltage sweep rate. Inset show $I-V$ curves in lower voltage region $(0-2.5 \mathrm{~V})$

PEDOT:PSS is at $5.2 \mathrm{eV}$ which is within the band gap of $\mathrm{ZnO}$ forming a Schottky junction at this interface.

The ideality factor $(n)$ and series resistance $\left(R_{s}\right)$ of Schottky contacts can be obtained from following equations using the Cheung method [31]:

$\frac{\mathrm{dV}}{\mathrm{d}(\ln \mathrm{I})}=\frac{\mathrm{nKT}}{\mathrm{q}}+\mathrm{IR}_{\mathrm{s}}$

$H(I)=V-\left(\frac{n k T}{q}\right) \ln \left(\frac{I}{A A * T^{2}}\right)$

$H(I)=n \phi_{b}+I R_{s}$

Figure 6 shows variation of $\mathrm{d} V / \mathrm{d}(\ln I)$ versus $I$ at different temperatures from which the value of $n$ can be obtained from the intercept at the zero current axis equating to $(n k T / q)$ in a linear fit of lower voltage region I. The value of $n$ is observed from the inset of Fig. 6 to decrease from 6.6 at $218 \mathrm{~K}$ to 2.2 at $298 \mathrm{~K}$. A similar pattern of variation of $n$ from 7.55 to 1.77 is observed for $\mathrm{Pd} / \mathrm{ZnO}$ Schottky barrier diodes as $\mathrm{T}$ is varied from 60 to $300 \mathrm{~K}$ [32]. These values are higher than the ideal value of $n=1-2$ for homogenous $\mathrm{ZnO}$ p-n junctions [33, 34]. Two different possible mechanisms are believed to be responsible depending upon the temperature: (i) generation recombination for $\mathrm{T} \leq 260 \mathrm{~K}$ and (ii) thermionic emission from $260 \mathrm{~K}<\mathrm{T} \leq 300 \mathrm{~K}$. However other factors that may contribute to the deviation of $\mathrm{n}$ from unity are current leakage effects, a lack of free-carrier concentration at low temperatures, parasitic rectifying junctions within the device and Schottky barrier inhomogeneity in the junction area [35]. Parameter $H(I)$ can be calculated using ' $n$ ' in Eq. 5. Figure 7 shows variation of $H(I)$ with current $(I)$ at three temperatures and the values of $\phi_{b}$ and $R_{s}$ have

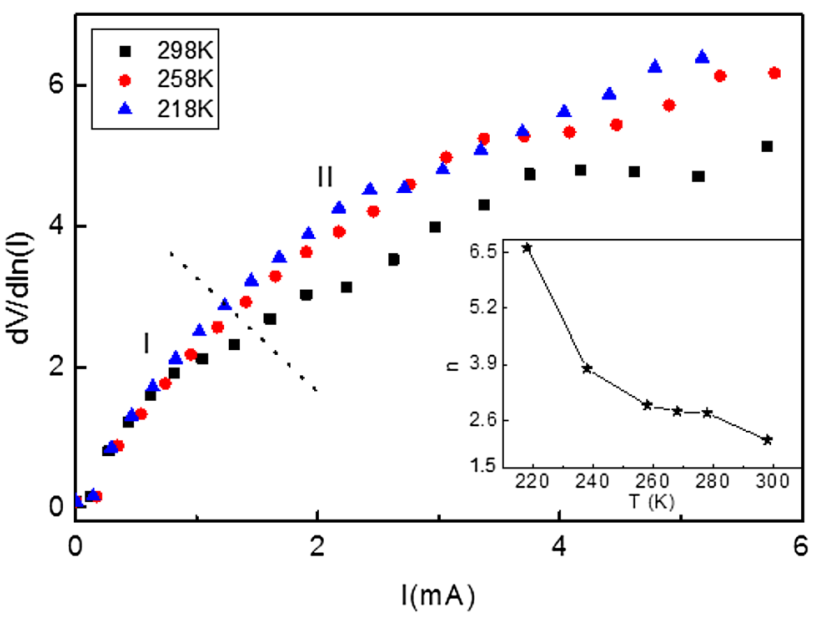

Fig. 6 Variation of $\mathrm{d} V / \mathrm{d} \ln (I)$ with $I$ for FTO/ZnO/PEDOT:PSS device at different temperatures in forward bias. Inset shows variation of $n$ with temperature

been estimated from the slope and intercept of the linear fit, respectively.

Figure 8 shows the variation of $R_{s}$ and $\phi_{b}$ with temperature T for FTO/ZnO/PEDOT:PSS Schottky junctions. $R_{s}$, the important parameters of electrical characterization plays a crucial role of non-ideal behaviour of forward bias current due to the presence of an interface layer between $\mathrm{ZnO}$ and the polymer. The decrease in $R_{s}$ with an increase in temperature may be due to barrier modification under the influence of temperature. This may be one of the reasons for the increase in $n$ at low temperature as shown in inset of Fig. 6 . The magnitude of barrier height $\varphi_{b}$ of the device is 0.78 at room temperature. If we establish forward bias conditions by applying a positive voltage to the $n$-type material, the majority carrier, electrons, will be attracted to the interface,

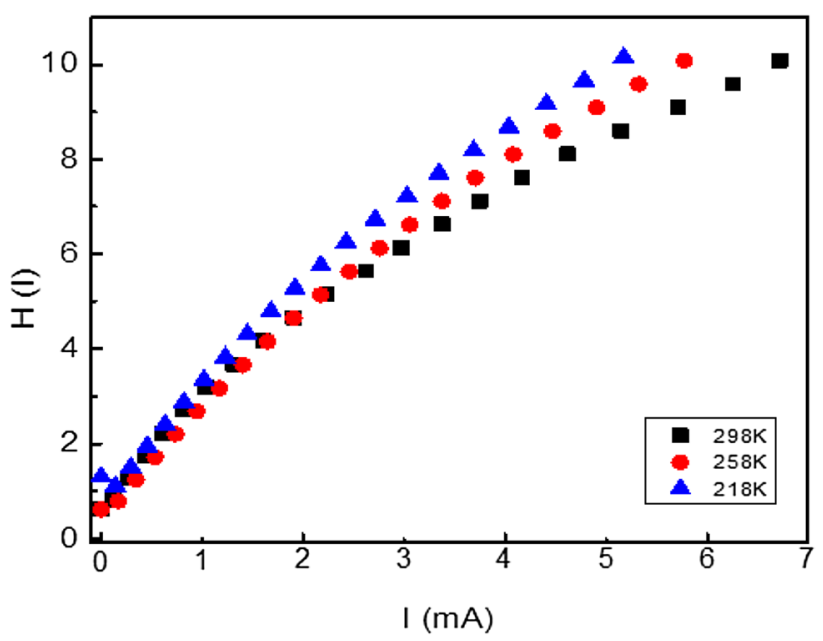

Fig. 7 Variation of $\mathrm{H}(I)$ with $I$ for FTO/ZnO/PEDOT:PSS device at different temperatures in forward bias 

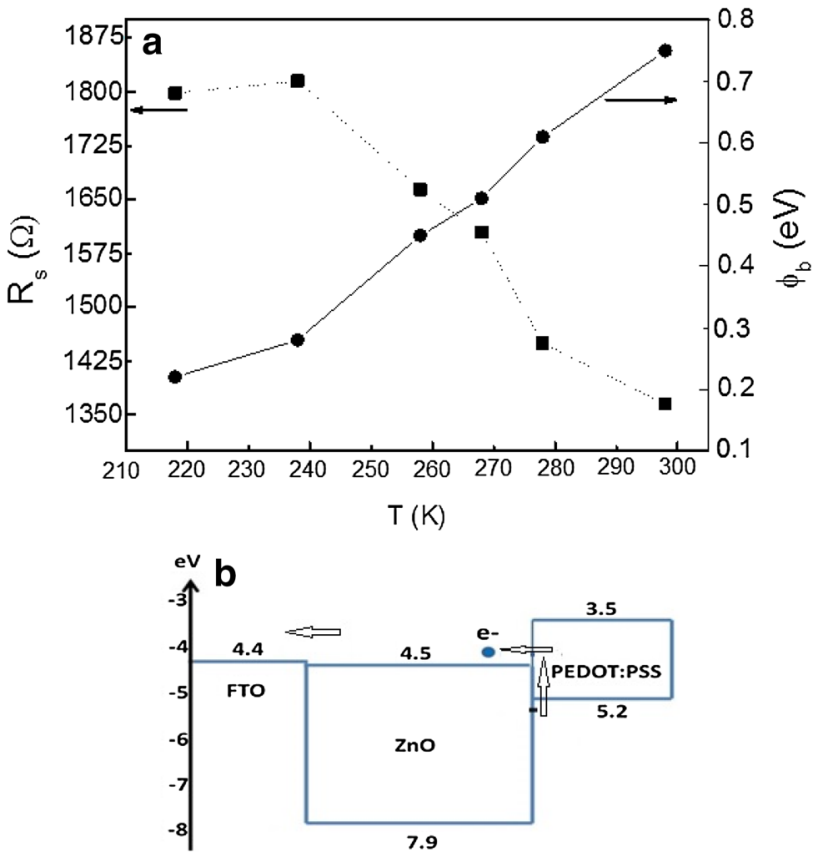

Fig. 8 a Variation of $R_{s}$ (left axis) and barrier height $\left(\varphi_{b}\right)$ (right axis) with temperature for $\mathrm{FTO} / \mathrm{ZnO} / \mathrm{PEDOT}$ :PSS device in forward bias obtained from $\mathrm{H}(I)$ function versus $I$ plot. b Shows band diagram and charge transport directions inside the device

reducing the depletion width to near zero. For $p$-type materials, a negative voltage is required to accumulate majority carrier holes. To delineate carrier type behaviour, the FTO/ZnO/PEDOT:PSS device structure was fabricated with both $n$-type $\mathrm{ZnO}$ and $p$-type PEDOT:PSS materials. Without applying any bias, $\varphi_{b}$ for electrons and holes are 1.1 and $5.7 \mathrm{eV}$ respectively as shown in band diagram of the device in the inset of Fig. 8b. The experimental data suggest that the majority carriers in this case are electrons, which accumulate near the ZnO/PEDOT:PSS Schottky junction at room temperature. The decrease in $\phi_{b}$ with lowering the temperature may be due to an inhomogeneous barrier interface between $\mathrm{ZnO}$ and PEDOT:PSS. Charge carriers at low temperature are able to cross low barriers and current transport across the interface would be caused by current flowing through patches of lower Schottky barrier height [36]. The number of charge carriers with sufficient energy to overcome the barriers would be expected to increase with an increase in temperature. Therefore, current transport through lower Schottky barrier height areas and a larger ideality factor would tend to dominate device characteristics.

The presence of a possible space charge limited current (SCLC) transport mechanism in the device has also been explored. The high value of $n$, presence of large series resistance and a low zero-bias barrier height indicate that thermionic emission is not the only current transport mechanism operational at higher voltages (region II). In this region, the behaviour of the forward bias current is typical of a SCLC transport mechanism, controlled by trap levels. The relation for the current $I$ in this case is given by following equation [37]:

$I \infty\left(\frac{9}{8}\right) \epsilon \epsilon_{0} a \mu \frac{V^{2}}{d^{3}}$

where $V$ is the applied voltage, $\mu$ is the carrier mobility, $\varepsilon=9$ is the dielectric constant of $\mathrm{ZnO}, a$ is the active area and $d$ is the distance between the electrodes $(5169 \mathrm{~nm})$. When the applied voltage is low, the current appears to be controlled by thermally generated carriers, rather than injected carriers. However, once the bias voltage is exceeded the turn-on voltage, injected carriers dominate over the thermally generated carriers. Further increases in applied voltage results in the formation of a space charge region formed from injected carriers in the bulk of wide band gap semiconductor. Further carrier injection is then inhibited by the presence of injected space charge by increasing applied voltage $(V>3 \mathrm{~V})$, giving
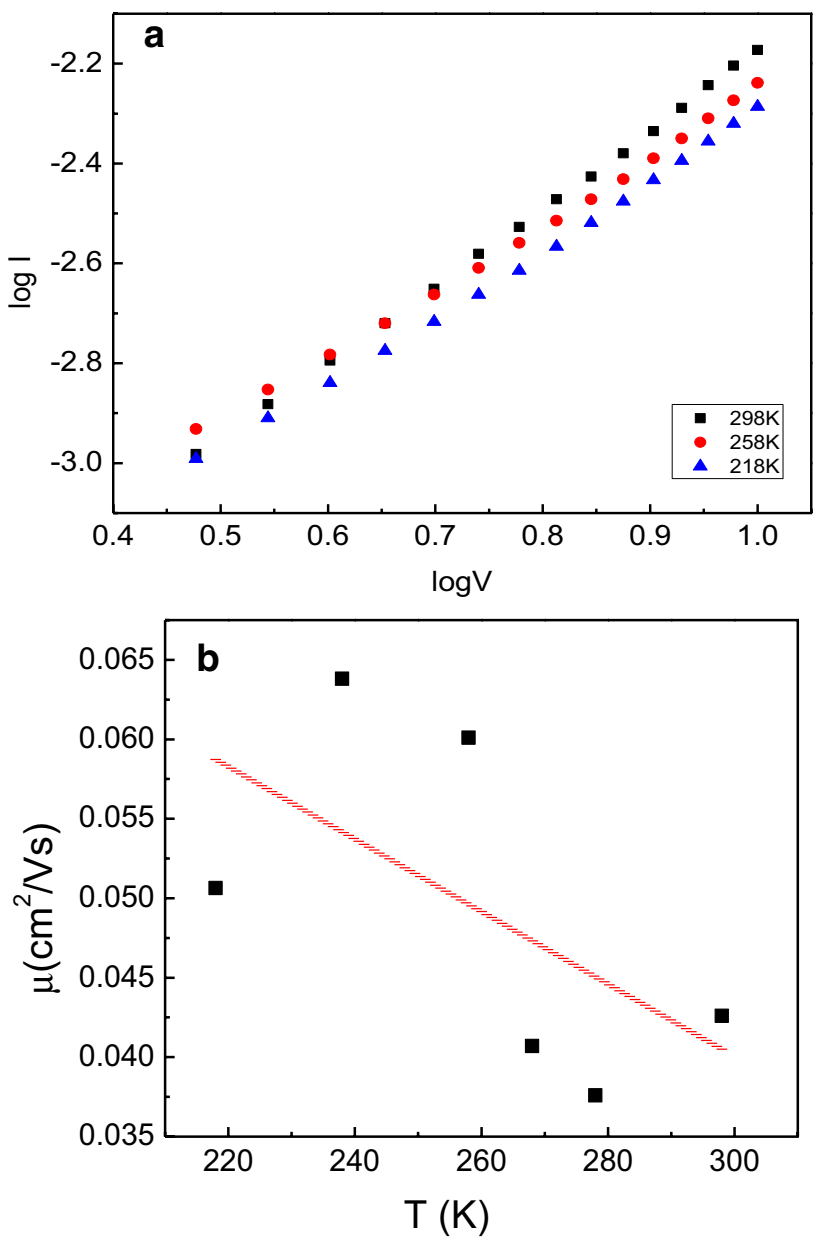

Fig. 9 a Variation of $\log I$ with $\log V$ plot for higher voltage region II of FTO/ZnO/PEDOT:PSS device at different temperatures. b Shows variation of mobility of charge carriers $(\mu)$ with temperature 


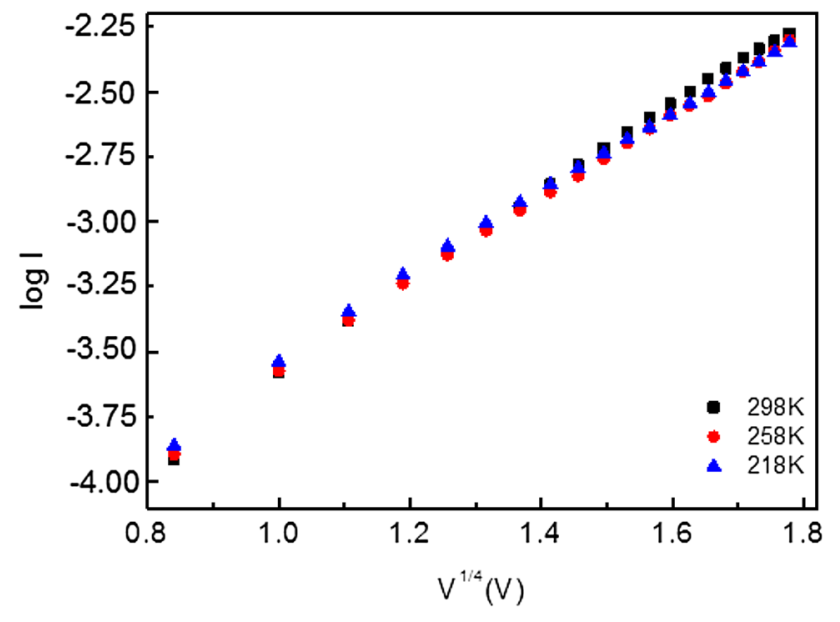

Fig. 10 Variation of $\log (I)$ with $\mathrm{V}^{1 / 4}$ for FTO/ZnO/PEDOT:PSS device in reverse bias at different temperatures rise to $I \sim V^{\mathrm{m}}$ limited conduction. Charge carrier mobility $\mu$ can be estimated from Eq. 7 for the device, accounting its geometry, for all temperatures. Figure 9 shows a plot of the variation of $\log I$ with $\log V$ at different temperatures, for the higher voltage region II of the device, following Eq. 7. The maximum $\mu$ obtained, from the intercept of linear plot between $\log I$ versus $2 \log V$ at room temperature, is $0.0426 \mathrm{~cm}^{2} /$ Vs. Similar SCLC has been reported for $330 \mathrm{~nm}$ thick $\mathrm{ZnO}$ film with $10 \%$ polymethyl vinyl etheralt-maleic anhydride stabilisers on glass substrates at room temperature under atmospheric condition [38]. The inset of Fig. 9 shows the magnitude of $\mu$ decreases with an increase in temperature, which may for example be caused by changes in surface states of polymer related to specific surface groups.

$I-V$ curves in reverse bias, as shown in Fig. 4, show a lack of saturation of the current with voltage which indicates that the Schottky barrier height may be bias dependence. There are many reasons for this dependence on image force,

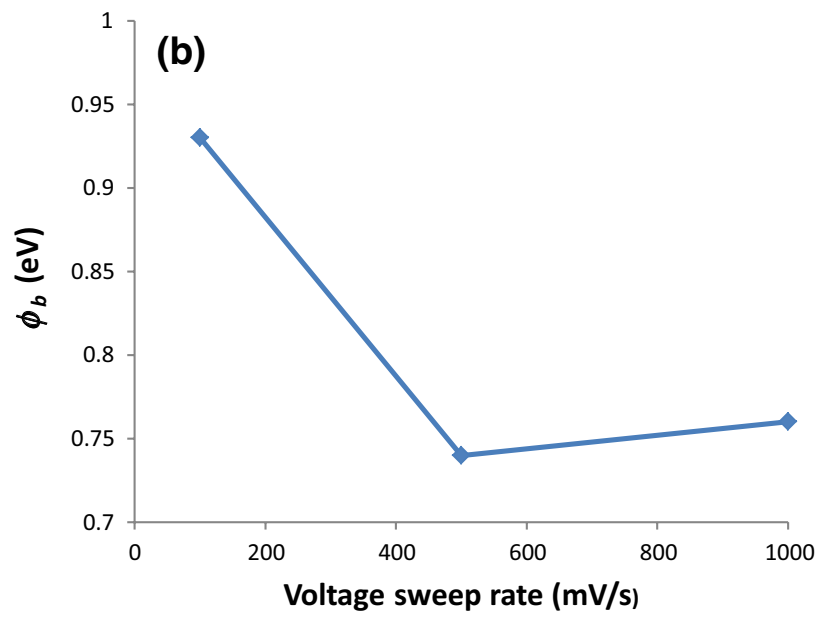

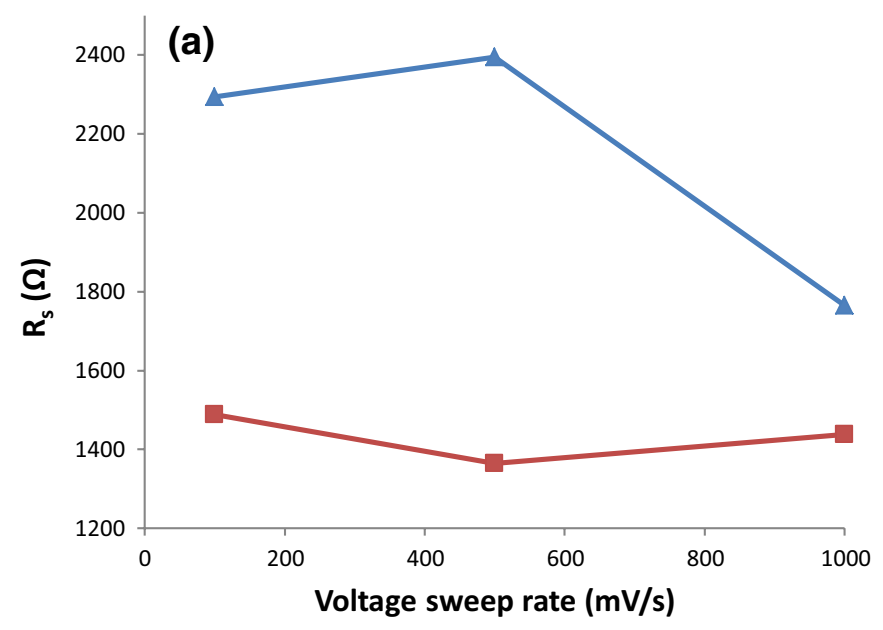

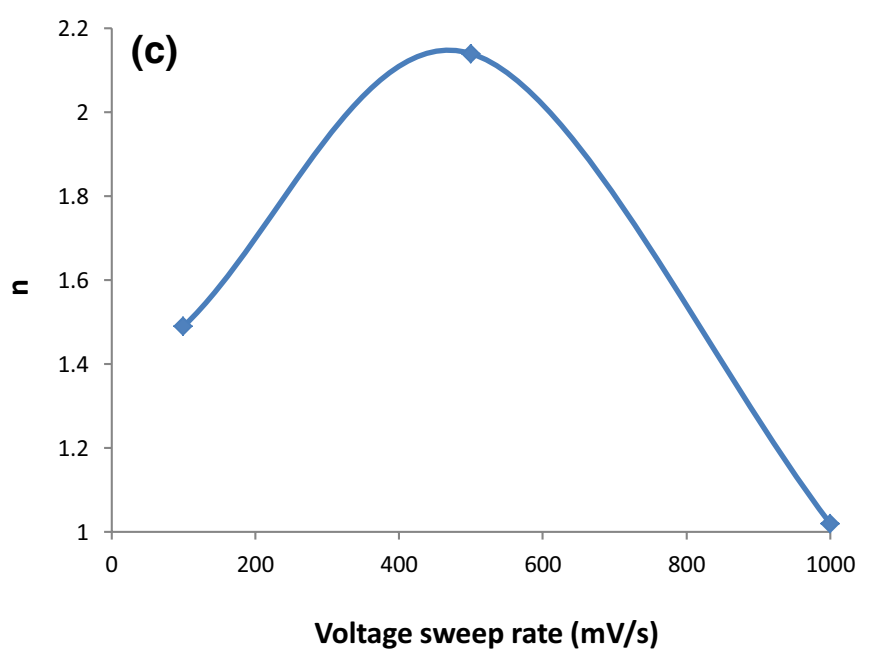

Fig. 11 a Series resistance $\left(R_{s}\right)$, calculated from Equation of 4 (solid triangle) and Equation of 6 (solid square), b Schottky barrier height $\left(\varphi_{b}\right)$ and $\mathbf{c}$ ideality factor $(n)$ 
interfacial layers and barrier inhomogeneities [39]. The barrier height lowering $\left(\Delta \phi_{b}\right)$ due to image force effect alone could be checked by plotting a graph between $\log I$ versus reverse bias voltage $V_{r}^{1 / 4}$ as shown in Fig. 10. The straight line nature of this plot confirms the effect of image force on Schottky barrier height which is also supported by studies published in the literature [40]. Further measurements have been performed at room temperature to examine the effect of the scan rate on the Schottky barrier height $\phi_{b}$, ideality factor $n$ and series resistance $R_{s}$. The dependences of these Schottky diode parameters on the voltage sweep rate are shown in Fig. 11 as the bias voltage was swept from +10 to $0 \mathrm{~V}$ with the rate varying from 100 to $1000 \mathrm{mV} / \mathrm{s}$. It can be seen that the diode behaviour is nearly ideal at $1000 \mathrm{mV} / \mathrm{s}$ in relative to lower scan rates. The accuracies $\Delta R_{s}$ in values of $R_{s}$ are found to be significantly improved when Eq. (6) is used instead of Eq. (4) and $\Delta R_{S}$ is estimated to be small as $\pm 8 \Omega$.

\section{Conclusions}

In this work, $\mathrm{FTO} / \mathrm{ZnO} / \mathrm{PEDOT}$ :PSS devices have been prepared by a simple spin-coating method. The morphological, spectroscopic and optical properties of the $\mathrm{ZnO}$ annealed film has been investigated with XRD, FTIR, and Photoluminescence measurements respectively. The electrical properties of this Schottky device have also been studied in depth with the help of DC $I-V$ measurements in the temperature range of 218-298 K. Voltage sweep rate independent, hysteresis-free $I-V$ plot shows the rectifying nature of the device which may result from the electrical nature of the components rather than from interface or contact related phenomena. The parameters such as $\varphi_{b}, n, R_{s}$ of this structure have been calculated from the forward bias $I-V$ characteristics as a function of device temperature following Cheung's method in the lower voltage region I. The value of $\varphi_{b}$ was found to increase with increases in temperature whereas the values of $n$ and $R_{s}$ decrease with temperature. In the higher voltage region II, the SCLC conduction mechanism is applicable for the device and temperature dependent mobility values have been calculated. In summary, it has been shown that the properties of FTO/ZnO/PEDOT:PSS structures, prepared by a simple spin-coating method, are suitable for use as highperformance Schottky diode devices. Hence PEDOT:PSS could be successfully used as an alternative to expensive metal electrodes in $\mathrm{ZnO}$-based inorganic semiconductor devices.

Acknowledgements This work is sponsored by the Air Force Office of Scientific Research, Air Force Material Command, USAF, under Grant No. FA8655-08-1-3056.
Open Access This article is distributed under the terms of the Creative Commons Attribution 4.0 International License (http://creativecommons.org/licenses/by/4.0/), which permits unrestricted use, distribution, and reproduction in any medium, provided you give appropriate credit to the original author(s) and the source, provide a link to the Creative Commons license, and indicate if changes were made.

\section{References}

1. Y. Xia, K. Sun, J. Ouyang, Solution-processed metallic conducting polymer films as transparent electrode of optoelectronic devices. Adv. Mater. 24, 2436-2440 (2012)

2. F.E. Jones, B.P. Wood, J.A. Myers, C. Daniels-Hafer, M.C. Lonergan, Current transport and the role of barrier inhomogeneities at the high barrier n-InP I poly(pyrrole) interface. J. Appl. Phys. 86, 6431-6441 (1999)

3. S. Kee, N. Kim, B.S. Kim, S. Park, Y.H. Jang, S.H. Lee, J. Kim, J. Kim, S. Kwon, K. Lee, Controlling molecular ordering in aqueous conducting polymers using ionic liquids. Adv. Mater. 28(39), 8625-8631 (2016). https://doi.org/10.1002/adma.201505473

4. N. Kim, S. Kee, S.H. Lee, B.H. Lee, Y.H. Kahng, Y.R. Jo, B.J. Kim, K. Lee, Highly conductive PEDOT:PSS nanofibrils induced by solution-processed crystallization. Adv Mater. 26, 2268-2272 (2014)

5. H. Xu, R.F. Chen, Q. Sun, W.Y. Lai, Q.Q. Su, W. Huang, X.G. Liu, Recent progress in metal-organic complexes for optoelectronic applications. Chem. Soc. Rev. 43(10), 3259-3302 (2014). https://doi.org/10.1039/c3cs60449g

6. J.Y. Kim, J.Y. Oh, S. Cheon, H. Lee, J. Lee, J.I. Lee, H. Ryu, S.M. Cho, T.Y. Kim, C.S. Ah, Y.H. Kim, C.S. Hwang, Optimized ion diffusion depth for maximizing optical contrast of environmentally friendly PEDOT:PSS electrochromic devices. Opt. Mater. Express 6(10) 3127-3134 (2016). https://doi.org/10.1364/OME.6.003127

7. M. Cai, Z. Ye, T. Xiao, R. Liu, Y. Chen, R.W. Mayer, R. Biswas, K.-M. Ho, R. Shinar, J. Shinar, Extremely efficient indium-tinoxide-free green phosphorescent organic light-emitting diodes. Adv. Mater. 24, 4337-4342 (2012)

8. S.H. Park, J. Kim, C.E. Park, J. Lee, H.S. Lee, S. Lim, S.H. Kim, Optimization of electrohydrodynamic-printed organic electrodes for bottom-contact organic thin film transistors. Org. Electron. 38, 48-54 (2016)

9. Y.F. Wang, B.Y. Jia, F. Qin, Y. Wu, W. Meng, S.X. Dai, Y.H. Zhou, X.W. Zhan, Semi-transparent, non-fullerene and flexible all-plastic solar cells. Polymer 107 108-112 (2016)

10. C.F. Zhang, T.M. Higgins, S.H. Park, S.E. O'Brien, D.H. Long, J. Coleman, V. Nicolosi, Highly flexible and transparent solid-state supercapacitors based on RuO2/PEDOT:PSS conductive ultrathin films. Nano Energy 28, 495-505 (2016). https://doi.org/10.1016/j. nanoen.2016.08.052

11. L.J. Brillson, Y. Lu, ZnO Schottky barriers and Ohmic contacts. J. Appl. Phys. 109, 121301 (2011)

12. M. Nakano, A. Tsukazaki, R.Y. Gunji, K. Ueno, A. Ohtomo, T. Fukumura, M. Kawasaki, Schottky contact on a $\mathrm{ZnO}(0001)$ single crystal with conducting polymer. Appl. Phys. Lett. 91, 142113 (2007)

13. M. Nakano, T. Makino, A. Tsukazaki, K. Ueno, A. Ohtomo, T. Fukumura, H. Yuji, S. Akasaka, K. Tamura, K. Nakahara, T. Tanabe, A. Kamisawa, M. Kawasaki, Transparent polymer Schottky contact for a high performance visible-blind ultraviolet photodiode based on ZnO. Appl. Phys. Lett. 93, 123309 (2008)

14. R.Y. Gunji, M. Nakano, A. Tsukazaki, A. Ohtomo, T. Fukumura, M. Kawasaki, Polymer Schottky contact on O-polar ZnO with silane coupling agent as surface protective layer. Appl. Phys. Lett. 93, 012104 (2008) 
15. S. Paul, P.G. Harris, C. Pal, A.K. Sharma, A.K. Ray, Low cost zinc oxide for memristors with high on-off ratios. Mater. Lett. 130, 40-42 (2014)

16. S. Paul, P.G. Harris, C. Pal, A.K. Sharma, A.K. Ray, Study of dielectric relaxation processes in printable zinc oxide films on transparent substrates. J. Mater. Sci. 26(9) 7109-7116 (2015). https://doi.org/10.1007/s10854-015-3333-4

17. N. Hernandez-Como, A. Rodriguez-Lopez, F.J. Hernandez-Cuevas, J. Munguia, R. Garcia, R. Baca-Arroyo, M. Aleman, Currentvoltage-temperature characteristics of PEDOT:PSS/ZnO thin filmbased Schottky barrier diodes. Semicond. Sci. Technol. (2016). https://doi.org/10.1088/0268-1242/31/11/115007

18. J.T. Wang, X.L. Shi, W.W. Liu, X.H. Zhong, J.N. Wang, L. Pyrah, K.D. Sanderson, P.M. Ramsey, M. Hirata, K. Tsuri, Influence of preferred orientation on the electrical conductivity of fluorinedoped tin oxide films. Sci. Rep. 4, 3679 (2014)

19. B.D. Cullity, S.R. Stock, Elements of X-ray Diffraction, 3rd edn. (Prentice-Hall, Englewood Cliff, 2001)

20. F.V. Molefe, L. Koao, F. Dejene, B.F. Swart H C, Phase formation of hexagonal wurtzite $\mathrm{ZnO}$ through decomposition of $\mathrm{Zn}(\mathrm{OH})(2)$ at various growth temperatures using CBD method. Opt. Mater. 46, 292-298 (2015). https://doi.org/10.1016/j. optmat.2015.04.034.

21. N.G. Shimpi, S. Jain, N. Karmakar, A. Shah, D.C. Kothari, S. Mishra, Synthesis of $\mathrm{ZnO}$ nanopencils using wet chemical method and its investigation as LPG sensor. Appl. Surf. Sci. 390, 17-24 (2016). https://doi.org/10.1016/j.apsusc.2016.08.050

22. S.D. Birajdar, P.P. Khirade, T.S. Saraf, R.C. Alange, K.M. Jadhav, Sol-gel auto combustion synthesis, electrical and dielectric properties of $\mathrm{Zn} 1-\mathrm{xCoxO}(0.0<=\mathrm{x}<=0.36)$ semiconductor nanoparticles. J. Alloys Compd. 691, 355-363 (2017). https:// doi.org/10.1016/j.jallcom.2016.08.220

23. M. Benhaliliba, C.E. Benouis, M.S. Aida, F. Yakuphanoglu, A.S. Juarez, Indium and aluminium-doped $\mathrm{ZnO}$ thin films deposited onto FTO substrates: nanostructure, optical, photoluminescence and electrical properties. J. Sol-Gel Sci. Technol. 55(3), 335-342 (2010)

24. K. Vanheusden, W.L. Warren, C.H. Seager, D.R. Tallant, J.A. Voigt, B.E. Gnade, Mechanisms behind green photoluminescence in zno phosphor powders. J. Appl. Phys. 79, 7983-7990 (1996)

25. U.P. Shaik, P.A. Kumar, M.G. Krishna, S.V. Rao, Morphological manipulation of the nonlinear optical response of $\mathrm{ZnO}$ thin films grown by thermal evaporation. Mater. Res. Express (2014). https://doi.org/10.1088/2053-1591/1/4/046201.

26. S.D. Kshirsagar, U.P. Shaik, M.G. Krishna, S.P. Tewari, Photoluminescence study of $\mathrm{ZnO}$ nanowires with $\mathrm{Zn}$ residue. J. Lumin. 136, 26-31 (2013)
27. G. Yip, J. Qiu, W.T. Ng, Z.H. Lu, Effect of metal contacts on the electrical characteristics of $\mathrm{Al}(2) \mathrm{O}(3)$ dielectric thin films. Appl. Phys. Lett. (2008). https://doi.org/10.1063/1.2903708.

28. D.K. Schroder, Semiconductor Material and Device Characterization, 2nd edn. (Wiley, New York, 1998)

29. S.M. Sze, Physics of Semiconductor Devices, 2nd edn. (Willey, New York, 1981), pp. 7-8.

30. H.V. Wenksten, E.M. Kaidashev, M. Lorentz, H. Hochmuth, G. Biehne, J. Lenzer, V. Gottscalch, R. Pickenhain, M. Grundmann, Lateral homogeneity of Schottky contacts on $n$-type $\mathrm{ZnO}$. Appl. Phys. Lett. 84, 79 (2004)

31. S.K. Cheung, N.W. Cheung, Extraction of Schottky diode parameters from forward current-voltage characteristics. Appl. Phys. Lett. 49, 85 (1986)

32. W. Mtangi, F.D. Auret, C. Nyamhere, P.J.J. van Rensburg, A. Chawanda, M. Diale, J.M. Nel, W.E. Meyer, The dependence of barrier height on temperature for Pd Schottky contacts on $\mathrm{ZnO}$. Physica B 404(22) 4402-4405 (2009). https://doi.org/10.1016/j. physb.2009.09.022

33. S. Yilmaz, E. Bacaksiz, İ Polat, Y. Atasoy, Fabrication and structural, electrical characterization of $\mathrm{i}-\mathrm{ZnO} / \mathrm{n}-\mathrm{ZnO}$ nanorod homojunctions. Curr. Appl. Phys. 12, 1326-1333 (2012)

34. Y.-W. Song, K. Kim, J.P. Ahn, G.-E. Jang, S.Y. Lee, Physically processed $\mathrm{Ag}$-doped $\mathrm{ZnO}$ nanowires for all- $\mathrm{ZnO} \mathrm{p}-\mathrm{n}$ diodes. Nanotechnology 20, 275606 (2009)

35. C. Yim, N. McEvoy, G.S. Duesberg, Characterization of graphene-silicon Schottky barrier diodes using impedance spectroscopy. Appl. Phys. Lett. (2013). https://doi.org/10.1063/1.4829140

36. M.A. Yıldırım, B. Güzeldir, A. Ates, M. Sag ${ }^{\top}$ lam, Temperature dependent current-voltage characteristics of the $\mathrm{Zn} / \mathrm{ZnO} / \mathrm{n}-\mathrm{Si} / \mathrm{Au}-$ $\mathrm{Sb}$ structure with $\mathrm{ZnO}$ interface layer grown on $\mathrm{n}$-Si substrate by SILAR method. Microelectron. Eng. 88, 3075-3079 (2011)

37. A.K. Ray, C.A. Hogarth, A critical-review of the observed electrical-properties of MIM devices showing VCNR. Int. J. Electron. 57(1), 1-77 (1984). https://doi.org/10.1080/00207218408938882

38. S. Bubel, N. Mechau, H. Hahn, R. Schmechel, Trap states and space charge limited current in dispersion processed zinc oxide thin films. J. Appl. Phys. 108, 124502 (2010)

39. E.H. Rhoderick, R.H. Williams, Metal-Semiconductor Contacts, 2nd edn. (Oxford Science Publications, Oxford, 1988)

40. B.L. Sharma, Metal-Semiconductor Schottky Barrier Junctions and Their Applications. (Plenum Press, New York, 1984) 